\title{
Neurocysticercosis in Pregnancy
}

\author{
Camille Webb, MD ${ }^{1}$ Mauricio La Rosa, MD² Gayle Olson, MD² Miguel Cabada, MD ${ }^{1}$ \\ ${ }^{1}$ Division of Infectious Disease, Department of Internal Medicine, \\ University of Texas Medical Branch, Galveston, Texas \\ 2 Division of Maternal Fetal Medicine, Department of Obstetrics and \\ Gynecology, University of Texas Medical Branch, Galveston, Texas \\ Address for correspondence Camille Webb, MD, Division of \\ Infectious Disease, Department of Internal Medicine of University of \\ Texas Medical Branch, 3111 Avenue Q., Galveston, TX 77555-0435 \\ (e-mail: cmwebbca@utmb.edu).
}

Am J Perinatol Rep 2018;8:e51-e56.

\begin{abstract}
Keywords

- neurocysticercosis

- Taenia solium

- pregnancy

The normal physiologic changes during pregnancy contribute to nutritional, metabolic, and immunologic adjustments, which can have an impact on the presentation of several diseases. New onset seizures during pregnancy and the postpartum can be attributed to several etiologies. Patient demographic data as well as personal and social histories are key in determining the etiology of new onset seizures. Neurocysticercosis (NCC), a commonly overlooked etiology, must be included in the differential diagnosis of patients with new onset seizures coming from NCC endemic areas. The diagnosis is based on a combination of clinical findings, exposure history, imaging, and serology. We present two cases of patients with NCC that became symptomatic during pregnancy or postpartum period. We will review the epidemiology, clinical manifestations, and management of NCC in pregnancy.
\end{abstract}

Taenia solium is endemic in low-income countries in Latin American, sub-Saharan Africa, and Asia including India and China. ${ }^{1,2}$ Cysticercosis is an infection by the larval stage of $T$. solium that affects both humans and pigs. Humans can become infected by the adult parasite (Taenia) and by the larval stage (cysticercus) by eating improperly cooked pork infected with larvae, or food contaminated with feces harboring Taenia eggs, respectively. Pigs are likewise infected after ingesting Taenia eggs. Cysts in human hosts are commonly acquired from a tapeworm carrier in the same household. $^{3}$ Neurocysticercosis (NCC), the central nervous system infection, is associated with nearly one-third of seizure disorders in endemic areas, and an estimated 50 million people worldwide have the infection. ${ }^{4}$

In the United States, 2\% of all emergency department visits for seizures are attributable to NCC. ${ }^{5}$ NCC is more common in immigrants or travelers from endemic areas, particularly from Latin America. ${ }^{5-7}$ A study by O'Keefe et al showed that $45 \%$ of subjects admitted to US hospitals due to NCC between 1998 and 2011 were women and 60\% were between 18 and 44 years old, coinciding with their child bearing age. ${ }^{5}$ Transmission of NCC has been reported within the United States through contact with immigrant workers with taeniasis. $^{8,9}$

received

June 14, 2017

accepted after revision

February 13, 2018
Obstetricians-gynecologists and general practitioners may encounter patients with NCC in their practice, particularly in areas with large immigrant populations. ${ }^{10,11}$ A recent survey among practicing physicians in this specialty suggested significant gaps in the knowledge about NCC epidemiology and transmission. ${ }^{12}$ Only $15 \%$ of the obstetriciangynecologists interviewed in that study were able to correctly identify the transmission route of cysticercosis and $35 \%$ knew how to make the diagnosis.

In this article, we report two cases of NCC during pregnancy and early postpartum that presented in two different settings and review the literature from developed and developing countries about this disease. We discuss aspects of the presentation, diagnosis, and management of NCC in pregnant women to inform the general practitioner.

\section{Cases}

\section{Case 1}

A 25-year-old female G7P4024 emigrated from Honduras to the United States during the 6th month of her pregnancy. She had a history of encephalitis 1 year prior, while in Honduras, which was treated with antibiotics for 10 days. She subsequently experienced one to two seizures like episodes

Copyright $\odot 2018$ by Thieme Medical Publishers, Inc., 333 Seventh Avenue, New York, NY 10001, USA. Tel: +1(212) 584-4662.
License terms

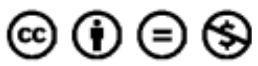

DOI https://doi.org/ 10.1055/s-0038-1639615. ISSN 2157-6998. 
monthly and intermittently took anticonvulsants during the pregnancy. A key component of her story is the personal, social, and family history. In Honduras, she worked in agriculture harvesting coffee beans and fruits. Her family practiced subsistence farming and raised free roaming pigs for consumption. A 7-year-old relative in the same household had recently been diagnosed with seizures.

She underwent an uncomplicated spontaneous vaginal delivery at term with a tubal ligation. However, on postpartum day 4 she complained of dizziness and shakiness of her right upper extremity followed by generalized seizure activity and loss of consciousness by postpartum day 6 . The patient was transferred to a tertiary care center.

On admission, her physical exam was unremarkable. The computed tomography (CT) scan of the brain showed scattered intracranial calcifications and the magnetic resonance imaging (MRI) showed scattered foci of T2/fluid-attenuated inversion recovery signal hyperintensity in the bilateral cerebral white matter. She was diagnosed with NCC and deemed to have nonviable parasites. The patient was prescribed divalproex sodium for seizure control.

\section{Case 2}

A 29-year-old female G6 P5005 at 17 weeks estimated gestational age presented to a rural clinic in Cusco-Peru with a headache. Evaluations were unremarkable and outpatient management was continued. Six days later when fatigue and anorexia accompanied a severe headache, the patient was admitted with concerns for sepsis. During observation, a witnessed tonic-clonic seizure prompted her transfer to a tertiary care center.

Again, the personal, social, and family history were a key component of her diagnosis. She lived in a rural community known to perform pork household husbandry, and she was a homemaker with five other children. Upon admission to the tertiary care center, she was noted to have tachycardia and neck stiffness. A white blood cell of 11,200 cells/ $\mu \mathrm{L}$ (93\% neutrophils), hemoglobin of $12.1 \mathrm{~g} / \mathrm{dL}$ (altitude adjustment $9.7 \mathrm{~g} / \mathrm{dL}$ ), platelets of 218,000 cells $/ \mu \mathrm{L}$, and an urinalysis was normal. An ultrasound showed a viable 17-week fetus. A brain CT scan showed hydrocephalus and other findings suggesting intraventricular NCC. No lumbar puncture was performed. She was treated with phenytoin, dexamethasone, and mannitol. A ventriculoperitoneal shunt was planned but before it could be placed she developed increasingly severe headaches, mental status changes, and had a cardiac arrest. In spite of heroic measures, both the mother and fetus succumbed to her disease within 21 days of the initial presentation of headache.

\section{Discussion}

The principles of management of NCC in pregnant women are similar to nonpregnant patients. However, considerations about the mother-fetus well-being and illnesses inherent to pregnancy pose challenges to the general practitioner. As illustrated by our cases, the presentation of NCC varies significantly between patients. The symptoms of NCC depend on the stage of infection, location of the cysts, and host response to the injury. Fully viable cystic lesions usually remain subclinical unless compression of adjacent structures triggers symptoms, while decaying stages (colloidal cysts) and dead cysts (granulomas/calcifications) are associated with perilesional inflammation and seizures. ${ }^{3}$ Parenchymal lesions are associated with seizures, ventricular lesions with hydrocephalus, and subarachnoid lesions with hydrocephalus and strokes. The presentation may vary from an incidental finding in an otherwise asymptomatic woman to lifethreatening increased intracranial pressure or intractable seizures.

In the general population, the most common symptoms of NCC are headaches and seizures. ${ }^{3}$ During pregnancy, focal and generalized seizures and headaches with increased intracranial pressure seem to be more common (-Table 1 ). Altered mentation, coma, and death of the mother and fetus have also been reported. ${ }^{10,11,13}$ The new onset seizure activity associated with NCC can be confused for pre-eclampsia and eclampsia particularly during the second half of pregnancy. ${ }^{11,14-16}$ The confusion between these two diagnosis is compounded by the fact that the prevalence of eclampsia is higher in low-income countries (6 to 157 cases per 10,000 deliveries) than in high-income countries (1.5-10 per 10,0000 deliveries $)^{17-20}$ and that other common symptoms of NCC such as visual changes and altered mental status are often attributed to pre-eclampsia and eclampsia. Headache, a very common symptom reported by almost $60 \%$ of young women, may be regarded as "normal" during pregnancy. ${ }^{21}$ A detailed history and physical exam, including travel history and past medical history, are crucial to arrive at the correct diagnosis in these settings.

The effects of pregnancy on the clinical course of NCC are unknown and have not been addressed in prospective studies. In the few case reports of NCC during pregnancy, most women had no prior history of NCC and presented with new onset seizures or increased intracranial pressure (-Table 1 ). An Indian case-control study compared pregnant women enrolled in an epilepsy registry with and without NCC. This study suggested an increase in seizure activity during pregnancy in 4 out of 30 participants with NCC. Although seizure control during pregnancy was significantly worse in women without NCC, the same group also had very poor seizure control before pregnancy. ${ }^{22}$ The local and systemic immune responses during pregnancy progressively shift toward a Tregulatory environment and decreased cellular immune response favoring immune tolerance. ${ }^{23}$ The same systemic T-regulatory environment has been documented in subjects with NCC and is believed to promote parasite survival. ${ }^{24}$ Inflammation around the decaying cysts or calcified lesions is fundamental for uncontrolled seizures to occur in NCC. Thus, the systemic immune response during pregnancy does not support the worsening of seizure activity during this period. However, pregnancy is not a generalized state of immunosuppression and some local components of the immune response may be enhanced (i.e., phagocytosis and monocyte and neutrophils numbers) possibly explaining changes around the cysticerci. ${ }^{25}$ Also, it is unclear if other 


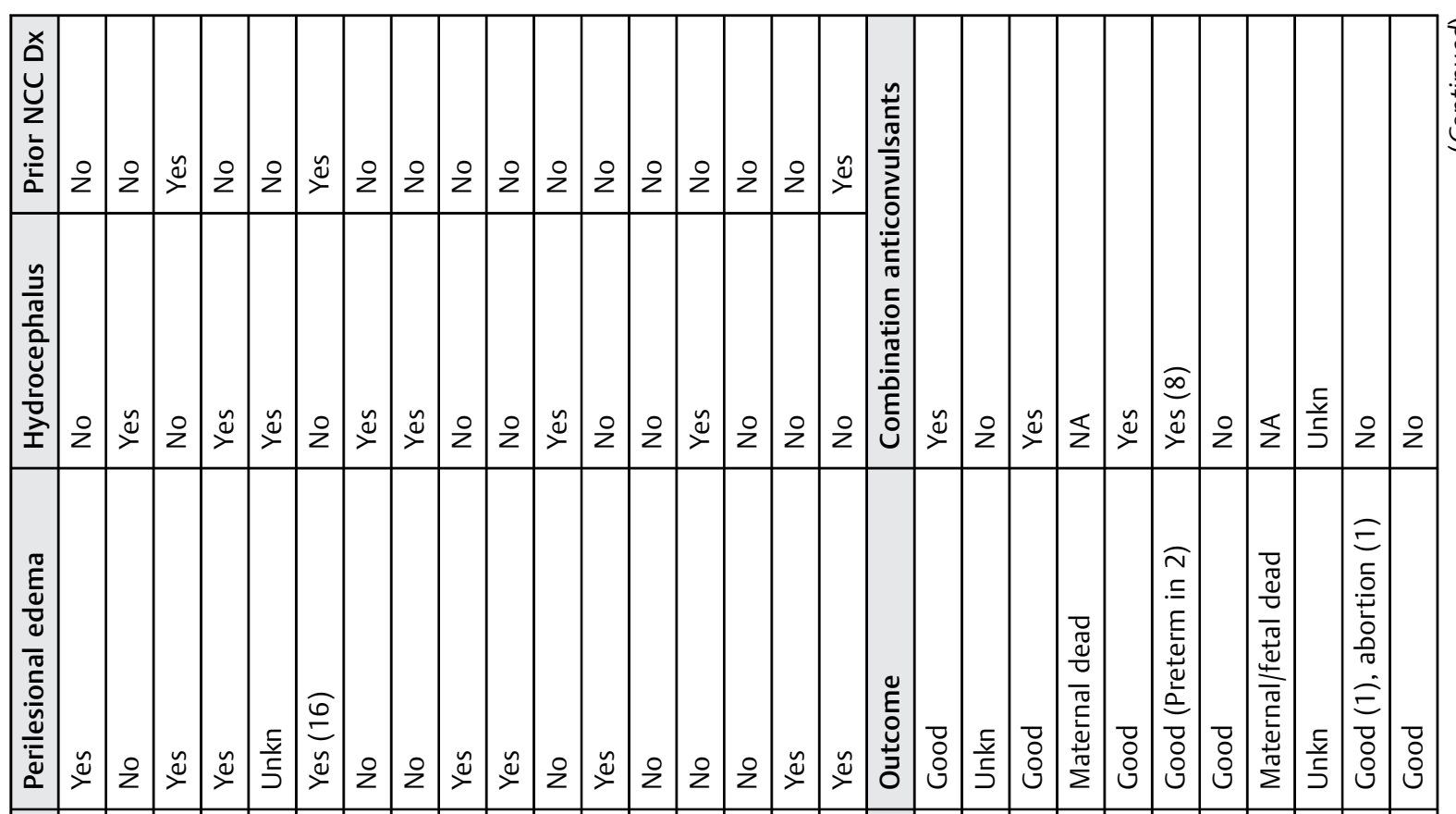

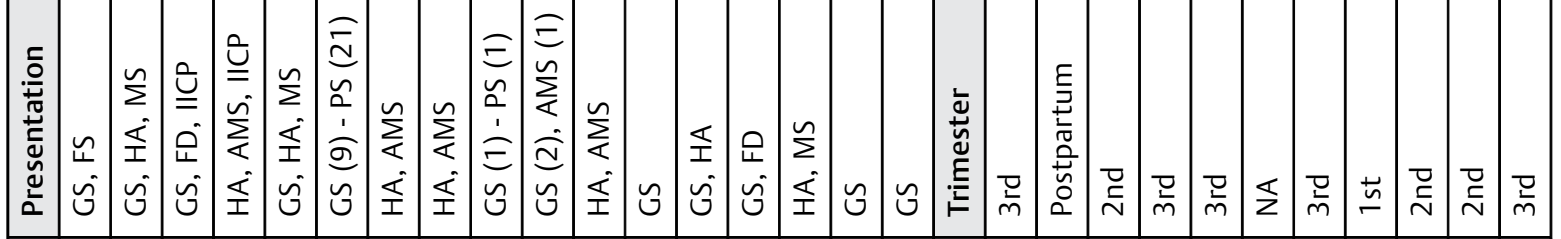

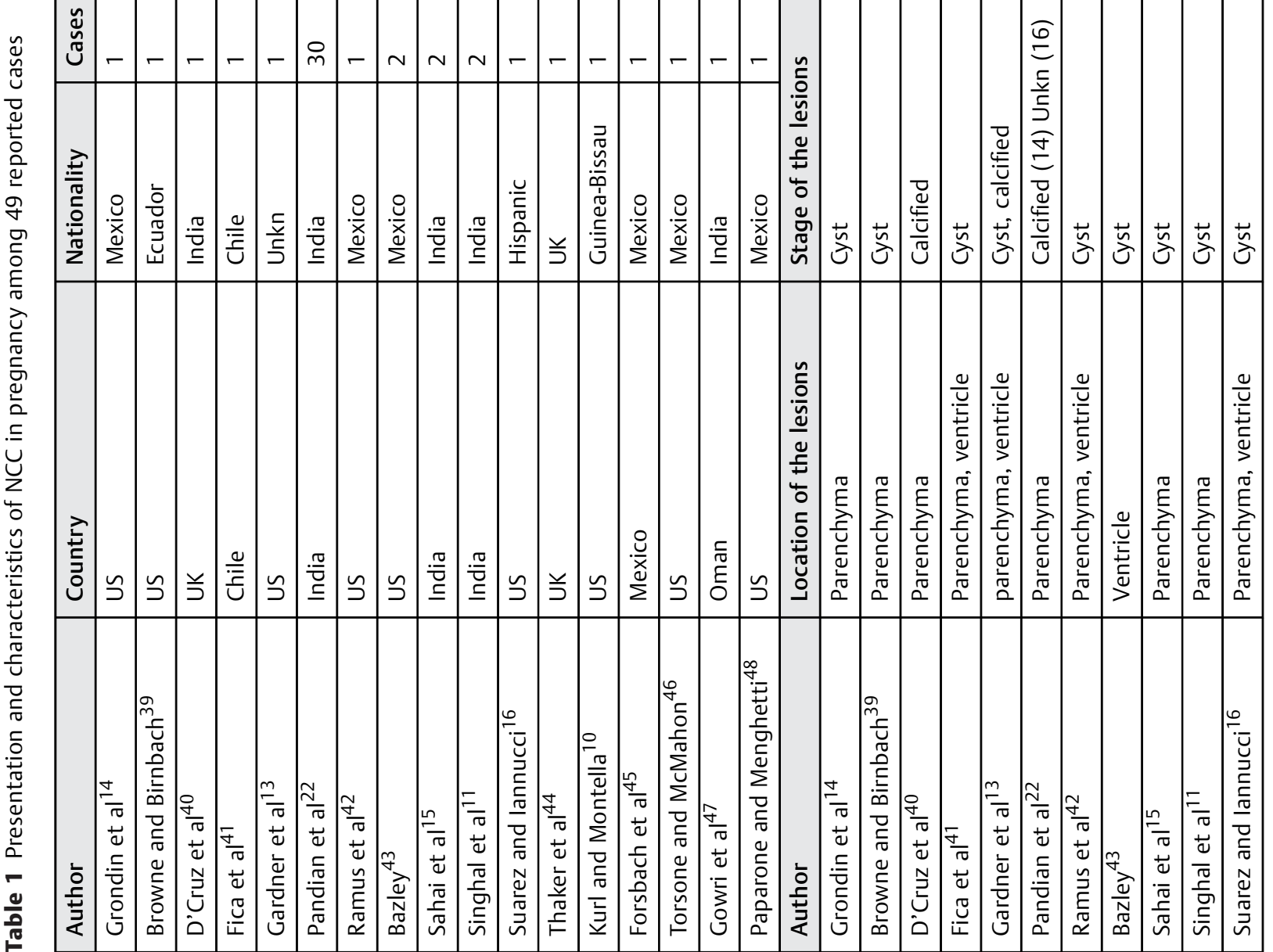


changes, like those seen during some gestational disorders in which inadequate response to angiogenic stimuli increase blood-brain barrier permeability, could explain an increase in seizure activity. ${ }^{26}$

There is no evidence that maternal infection with $T$. solium causes vertical transmission to the fetus. However, cysticercosis can be transmitted horizontally from the mother to the newborn and screening mothers with NCC for tapeworm infection is imperative. ${ }^{27}$ Most pregnancies in women with NCC are associated with good outcomes and there is no indication that maternal NCC itself causes fetal malformations or brain damage.(-Table 1) However, sustained seizures may pose a major risk to the fetus due to hypoxia. ${ }^{13}$

The diagnosis of NCC during pregnancy and postpartum is difficult and depends on a high level of suspicion. Eliciting the history of exposure in endemic areas as well as the personal, social, and family histories is the key for suspecting the diagnosis in patients with neurologic symptoms. The same criteria for the diagnosis of NCC in the general population apply in pregnancy. ${ }^{28}$ Imaging studies should be performed early when NCC is suspected in a pregnant woman. MRI is highly sensitive to identify small noncalcified cysts, edema, or enhancement around calcifications, and subarachnoid or intraventricular lesions. CT scan is superior to MRI for the evaluation of calcified lesions but has a lower sensitivity for subarachnoid or intraventricular lesions. MRI without gadolinium is not associated with teratogenicity and should be the test of choice during any stage of pregnancy. If CT scan is performed, narrow collimation, wide pitch, and shielding should be used.

The enzyme-linked immunoelectrotransfer blot (EITB) assay is the test of choice to detect antibodies to $T$. solium in serum. The sensitivity of EITB is very high for patients with more than one lesion, but only $50 \%$ to $60 \%$ for patients with single lesions. ELISA assays for the detection of antibodies are available but not recommended due to their low sensitivity and specificity. ${ }^{29}$ T. solium antigen detection is considered a major clinical/exposure diagnostic criteria. ${ }^{28,30,31}$ These tests may also be useful to monitoring the response to medical treatment. ${ }^{32,33}$

The management of NCC during pregnancy depends on the clinical presentation and should focus on symptomatic therapy. Most symptoms of NCC are associated with the host response to the parasite rather than infection itself. ${ }^{34,35}$ Antiparasitic therapy is associated with early worsening in seizure activity which should be avoided during pregnancy. While the safety of short courses of low-dose albendazole and praziquantel during pregnancy has been established in neglected tropical diseases control programs, the safety of prolonged high-dose treatment for NCC has not been studied. Benefits and risks of the antiparasitic therapy during pregnancy should be discussed with the patient, and in most cases therapy should be deferred until after delivery.

Women with new onset seizures between the second trimester and the postpartum will often be considered to have preeclampsia/eclampsia. However, in the absence of high blood pressure or other signs of preeclampsia, a different diagnosis must be suspected and neuroimaging ordered 
particularly for women at risk of transmission. Antiepileptic medication should be started to avoid seizure recurrence during pregnancy. Monotherapy is preferred since drug combinations, especially those containing valproic acid, are associated with a higher incidence of malformations when used in the first trimester. ${ }^{36}$ However, up to $16 \%$ of patients with NCC will not have adequate seizure control with only one agent. ${ }^{37}$ Corticosteroids are used to treat the inflammation associated with NCC lesions especially in patients with diffuse edema or mass effect from large cysticerci. Corticosteroids should be avoided in patients with calcified lesions due to worsening of symptoms after tapering of the medication. ${ }^{38}$ Patients with acute hydrocephalus should be evaluated for urgent surgical management to remove the parasite from the ventricles or to divert cerebrospinal fluid drainage. In pregnant and postpartum women with taeniasis, a single dose of the nonabsorbable antitapeworm medication niclosamide associated with a mild laxative should be prescribed to prevent cysticercosis transmission to the newborn.

\section{Conclusion}

NCC is one of the most common etiologies of seizures worldwide and should be considered in pregnant or postpartum women with compatible presentations, particularly if coming from endemic areas. Its diagnosis and management during pregnancy can be challenging, but prompt interventions are crucial for better maternal and fetal outcomes.

\section{References}

1 Coyle CM, Mahanty S, Zunt JR, et al. Neurocysticercosis: neglected but not forgotten. PLoS Negl Trop Dis 2012;6(05):e1500

2 Garcia H, Coyle C, White A Jr. Cysticercosis. In: Guerrant R, Walker D, Weller P, eds. Tropical Infectious Diseases, Principles, Pathogens and Practice. Philadelphia: Elsevier-Saunders; 2011: 815-823

3 Webb CM, White AC Jr. Update on the diagnosis and management of neurocysticercosis. Curr Infect Dis Rep 2016;18(12):44

4 Budke CM, White AC Jr, Garcia HH. Zoonotic larval cestode infections: neglected, neglected tropical diseases? PLoS Negl Trop Dis 2009;3(02):e319

5 Ong S, Talan DA, Moran GJ, et al; EMERGEncy ID NET Study Group. Neurocysticercosis in radiographically imaged seizure patients in U.S. emergency departments. Emerg Infect Dis 2002;8(06):608-613

6 O'Keefe KA, Eberhard ML, Shafir SC, Wilkins P, Ash LR, Sorvillo FJ. Cysticercosis-related hospitalizations in the United States, 19982011. Am J Trop Med Hyg 2015;92(02):354-359

7 O'Neal SE, Flecker RH. Hospitalization frequency and charges for neurocysticercosis, United States, 2003-2012. Emerg Infect Dis 2015;21(06):969-976

8 Centers for Disease Control (CDC). Locally acquired neurocysticercosis-North Carolina, Massachusetts, and South Carolina, 1989-1991. MMWR Morb Mortal Wkly Rep 1992;41(01):1-4

9 Moore AC, Lutwick LI, Schantz PM, et al. Seroprevalence of cysticercosis in an Orthodox Jewish community. Am J Trop Med Hyg 1995;53(05):439-442

10 Kurl R, Montella KR. Cysticercosis as a cause of seizure disorder in pregnancy: case report and review of literature. Am J Perinatol 1994;11(06):409-411

11 Singhal SR, Nanda S, Singhal SK. Neurocysticercosis as an important differential of seizures in pregnancy: two case reports. J Med Case Reports 2011;5:206
12 Dick GWA, Kitchen SF, Haddow AJ. Zika virus. I. Isolations and serological specificity. Trans R Soc Trop Med Hyg 1952;46(05): 509-520

13 Gardner E, Chang M, Mancuso P, Chaney SE. Neurocysticercosis in pregnancy: not just another headache. Nurs Womens Health 2012;16(02):118-124

14 Grondin L, D'Angelo R, Thomas J, Pan PH. Neurocysticercosis masquerading as eclampsia. Anesthesiology 2006;105(05): 1056-1058

15 Sahai S, Sahai A. Pica causing neurocysticercosis in pregnancy presenting as eclampsia: a report of two cases. J Obstet Gynaecol India 2013;63(01):68-69

16 Suarez VR, Iannucci TA. Neurocysticercosis in pregnancy: a case initially diagnosed as eclampsia. Obstet Gynecol 1999;93(5 Pt 2):816-818

17 Eke AC, Ezebialu IU, Okafor C. Presentation and outcome of eclampsia at a tertiary center in South East Nigeria-a 6-year review. Hypertens Pregnancy 2011;30(02):125-132

18 World Health Organization International Collaborative Study of Hypertensive Disorders of Pregnancy. Geographic variation in the incidence of hypertension in pregnancy. Am J Obstet Gynecol 1988;158(01):80-83

19 Liu S, Joseph KS, Liston RM, et al; Maternal Health Study Group of Canadian Perinatal Surveillance System (Public Health Agency of Canada). Incidence, risk factors, and associated complications of eclampsia. Obstet Gynecol 2011;118(05):987-994

20 Douglas KA, Redman CW. Eclampsia in the United Kingdom. BMJ 1994;309(6966):1395-1400

21 Aegidius K, Zwart JA, Hagen K, Stovner L. The effect of pregnancy and parity on headache prevalence: the Head-HUNT study. Headache 2009;49(06):851-859

22 Pandian JD, Venkateswaralu K, Thomas SV, Sarma PS. Maternal and fetal outcome in women with epilepsy associated with neurocysticercosis. Epileptic Disord 2007;9(03):285-291

23 Bonney EA. Immune regulation in pregnancy: a matter of perspective? Obstet Gynecol Clin North Am 2016;43(04):679-698

24 Arce-Sillas A, Álvarez-Luquín DD, Cárdenas G, et al. Interleukin 10 and dendritic cells are the main suppression mediators of regulatory $\mathrm{T}$ cells in human neurocysticercosis. Clin Exp Immunol 2016;183(02):271-279

25 Omer SB. Maternal immunization. N Engl J Med 2017;376(13): 1256-1267

26 Conti E, Zezza L, Ralli E, et al. Growth factors in preeclampsia: a vascular disease model. A failed vasodilation and angiogenic challenge from pregnancy onwards? Cytokine Growth Factor Rev 2013;24(05):411-425

27 Asnis D, Kazakov J, Toronjadze T, et al. Neurocysticercosis in the infant of a pregnant mother with a tapeworm. Am J Trop Med Hyg 2009;81(03):449-451

28 Del Brutto $\mathrm{OH}$, Nash TE, White AC Jr, et al. Revised diagnostic criteria for neurocysticercosis. J Neurol Sci 2017;372:202-210

29 Carod JF, Randrianarison M, Razafimahefa J, et al. Evaluation of the performance of 5 commercialized enzyme immunoassays for the detection of Taenia solium antibodies and for the diagnosis of neurocysticercosis. Diagn Microbiol Infect Dis 2012;72(01):85-89

30 Gabriël S, Blocher J, Dorny P, et al. Added value of antigen ELISA in the diagnosis of neurocysticercosis in resource poor settings. PLoS Negl Trop Dis 2012;6(10):e1851

31 Deckers N, Dorny P. Immunodiagnosis of Taenia solium taeniosis/ cysticercosis. Trends Parasitol 2010;26(03):137-144

32 Gonzalez AE, Bustos JA, Garcia HH, et al; Cysticercosis Working Group in Peru. Successful antiparasitic treatment for cysticercosis is associated with a fast and marked reduction of circulating antigen levels in a naturally infected pig model. Am J Trop Med Hyg 2015;93(06):1305-1310

33 Fleury A, Garcia E, Hernández M, et al. Neurocysticercosis: HP10 antigen detection is useful for the follow-up of the severe patients. PLoS Negl Trop Dis 2013;7(03):e2096 
34 Gonzales I, Rivera JT, Garcia HH; Cysticercosis Working Group in Peru. Pathogenesis of Taenia solium taeniasis and cysticercosis. Parasite Immunol 2016;38(03):136-146

35 Nash TE, Mahanty S, Loeb JA, et al. Neurocysticercosis: a natural human model of epileptogenesis. Epilepsia 2015;56(02):177-183

36 Crawford P. Epilepsy and pregnancy. Seizure 2002;11(Suppl A):212-219

37 Rajshekhar V, Jeyaseelan L. Seizure outcome in patients with a solitary cerebral cysticercus granuloma. Neurology 2004;62(12): 2236-2240

38 Mejia R, Nash TE. Corticosteroid withdrawal precipitates perilesional edema around calcified Taenia solium cysts. Am J Trop Med Hyg 2013;89(05):919-923

39 Browne IM, Birnbach DJ. Neurocysticercosis: a new differential in the diagnosis of postdural puncture headache. Anesth Analg 2003;97(02):580-582table of contents

40 D'Cruz RF, Ng SM, Dassan P. Neurocysticercosis in pregnancy: maternal and fetal outcomes. Oxf Med Case Rep 2016;2016(07): $138-140$
41 Fica C A, Castro S M, Soto S A, Flores M C, Oelker B C, Weitzel T. [Neurocysticercosis: an neglected disease in Chile]. Rev Chilena Infectol 2012;29(01):72-81

42 Ramus RM, Girson M, Twickler DM, Wendel GD. Acute obstructive hydrocephalus due to cysticercosis during pregnancy. Infect Dis Obstet Gynecol 1994;1(04):198-201

43 Bazley WS. Maternal mortality due to cysticercus cerebri. A case report. Obstet Gynecol 1972;39(03):362-367

44 Thaker HK, Tacconi L, Snow MH. Neurocysticercosis in pregnancy. Br J Neurosurg 2001;15(03):284

45 Forsbach G, Iris S, Zárate A, Azuela JC, Canales ES. Empty sella syndrome due to cysticercosis cerebri in a pregnant woman. Rev Invest Clin 1976;28(01):33-35

46 Torsone A, McMahon MJ. A guest editorial: neurocysticercosis. Obstet Gynecol Surv 2000;55(08):465-467

47 Gowri V, Jacob PC, Jain R, Sawhney S. Neurocysticercosis in pregnancy. Neurosciences (Riyadh) 2005;10(02):183-185

48 Paparone PW, Menghetti RA. Case report: neurocysticercosis in pregnancy. N J Med 1996;93(02):91-94 